(C) 1997 IEEE. Personal use of this material is permitted. Permission from IEEE must be obtained for all other uses, in any current or future media, including reprinting/republishing this material for advertising or promotional purposes, creating new collective works, for resale or redistribution to servers or lists, or reuse of any copyrighted component of this work in other works. 


\title{
PERFORMANCE OF DIGITAL COLLECTIVE ANTENNA SYSTEMS IN THE PRESENCE OF PHASE NOISE AND CLOCK JITTER
}

\author{
S. Merchán ${ }^{1}$, A. García Armada ${ }^{2}$ and J. L. García ${ }^{1}$ \\ ${ }^{1}$ University of Cantabria, Department of Communications Engineering, E.T.S.I.I. Telecomunicación, Spain \\ ${ }^{2}$ Polytechnical University of Madrid, Department of Signals, Systems and Radiocommunications, \\ E.T.S.I. Telecomunicación, Spain
}

\begin{abstract}
This paper studies the performance of digital collective systems in the presence of a noisy phase reference (phase noise) and a noisy clock reference (clock jitter). For this purpose, an attempt has been made to characterize the performance of this digital transmission system through the Bit Error Rate (BER) or Symbol Error Rate (SER) as a function of the Eb/No ratio. The Eb/No ratio has been parametrised by phase noise and clock jitter, obtained through computer simulations. It will be shown that these effects produce a significant increase in BER/SER performance. The simulator used was SPW (Signal Processing WorkSystem) by means of which BER/SER has been estimated using a quasianalytical (semianalytic) approach and the Monte Carlo method, so that the results can be compared and checked.
\end{abstract}

\section{INTRODUCTION.}

The introduction of digital TV could take place in three different scenarios: terrestrial broadcasting, satellite and cable networks. The latter two have already been approved under the standards DVB-S (Digital Video Broadcasting by Satellite) and DVB-C (Digital Video Broadcasting by Cable), respectively, and the terrestrial standard, DVB$\mathrm{T}$ (Digital Video Broadcasting by Terrestrial), will soon be ready.

SMATV (Satellite Master Antenna Television) systems can be considered as an optimal distribution network for a fast and effective introduction of digital TV to the end users in several European countries and is an alternative which can be used in those countries that are developing their infrastructures, since the key to the rapid introduction of digital TV is reaching the maximum number of users with the minimum change in the TV distribution infrastructures $[1],[2],[3]$.
Three types of systems for digital TV distribution through SMATV channel can be considered:

- SMATV-IF: The satellite QPSK signal, after being frequency down converted by the Low Noise Block (LNB) to IF band $(950-2050 \mathrm{MHz})$, is directly distributed on the SMATV network without any demodulation and re-modulation process at the head-end. The user requires a QPSK receiver as in the case of DTH (Direct to Home) reception.

- SMATV-S: The satellite QPSK signal, after being down converted by the LNB to $\mathrm{IF}$, is frequency converted again to extended S-band $(230-470 \mathrm{MHz})$. In this configuration, the satellite signal reaches the user IRD (Integrate Receiver Decoder) without any transmodulation process at the head-end. On the user's side, a frequency converter is used to restore the IF frequency and the signal is received by the DTH IRD.

- SMATV-DTM: The satellite QPSK signal is transmodulated to a 64-QAM scheme, as defined in the cable specification, DVB-C, in a transparent way. The concept of transparency means that the incoming signal from the satellite is not changed in content, but only a modulation change is applied in a very simple way in order to minimize the head-end costs. This configuration has the great advantage of using the same QAM IRD as that used by CATV networks, allowing the user to receive satellite and CATV signals with the same IRD . The spectrum usage is optimum since the signal from a satellite transponder, occupying between 27 and $36 \mathrm{MHz}$, is distributed in $7 / 8 \mathrm{MHz}$ channels. This is the most suitable choice. 
However, the SMATV-DTM configuration requires an interface between the satellite QPSK signal and the cable network's 64-QAM signal: the Transparent Digital Transmodulator (TDT). Its function is to change the satellite's QPSK modulation to the 64-QAM modulation of the cable network. During the conversion, error detection and correction processes are carried out, based on FEC (Forward Error Correction) coding (Reed Solomon, convolutional interleaving, convolutional encoding), in the QPSK signal. These processes are also incorporated in the generated 64-QAM signal.

A SMATV-DTM distribution system is shown in figure 1. The digital TV signal is broadcast by satellite using QPSK modulation. On the reception side, the signal is transmodulated to QAM at the head-end level and distributed to the users by the cable distribution network. The whole transmission chain is split into two sections, satellite and cable section, and there is an interface between them, the TDT, which adapts the signal from one section to the requirements of the other. A functional block diagram of the TDT is presented in figure 2.

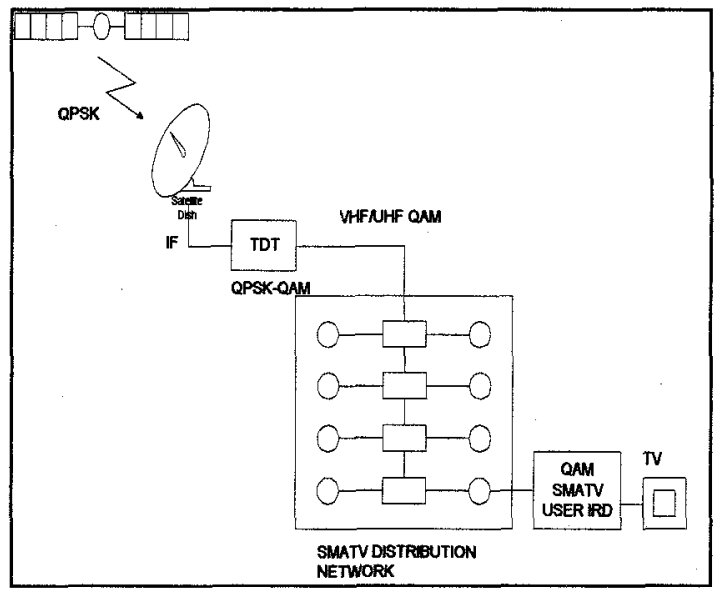

Figure 1. SMATV-DTM distribution system.

The designer of the TDT needs to know the tolerable rates of carrier phase noise and clock jitter for the later design or improvement of its subcircuits. Therefore, a SMATV-DTM system has been simulated with the aim of observing the increase in BER/SER as a function of a reference noisy carrier (phase noise) and as a function of the sample/decide clock sample point fluctuations, in the presence of additive white gaussian noise (AWGN).

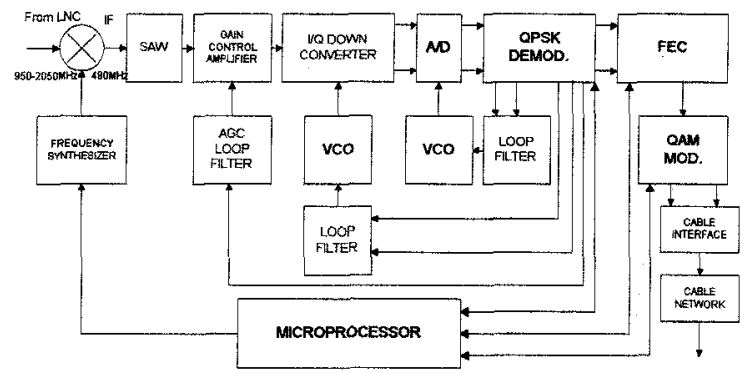

Figure 2. TDT functional block diagram.

In order to characterize the SMATV channel, an extensive measurement campaign has been carried out, covering all usual types of network configurations and using material from a great variety of manufacturers from different countries, on different kinds of old and new installations [4], [5], [6]. SMATV networks with all types of topologies and various different taps have been measured. A certain number of each type was selected, with the number of floors varying from 5 to 10 , and measurements were made at all the outlets.

The types of topology and tap of the measured networks are the following:

- New networks:
A.- Cascaded/Inductive.
B.- Cascaded/Resistive.
C. - Parallel/Inductive.
D.- Parallel/Resistive.

- Old networks:

E.- Cascaded/Inductive.

F.- Cascaded/Resistive.

G.- Parallel/Resistive.

The low percentage of old networks of the parallel/inductive type, has prevented its analysis. Networks less than 5 years old were considered to be new; the rest were classified as old. These measurements allowed us to characterize the SMATV channel, providing a reference for testing QAM equalizers for SMATV [6].

In the same way, computer simulations have been carried out where the performance of these systems is studied through the 
BER/SER curves as a function of the $\mathrm{Eb} / \mathrm{No}$ ratio [6], [7]. However, in these simulations an ideal carrier, synchronized in frequency and phase, with perfect timing recovery, is assumed. One of the most important aspects to bear in mind for characterizing these systems, consists of establishing the system quality degradation as a function of carrier-phase noise and clock jitter.

\section{SIMULATION RESULTS.}

The performance of these digital transmission systems is characterized by plotting BER/SER curves as a function of $\mathrm{Eb} /$ No ratio. However, the results presented in the literature so far [6], have assumed an ideal carrier and clock.

In the present work we have performed computer simulations for QPSK and 64-QAM digital transmission systems, in order to study how BER/SER is affected by a noisy phase reference and a noisy clock reference.

Phase noise is composed primarily of two contributions: tracked thermal noise and oscillator instability (untracked). The probability density function (pdf) that characterizes the phase noise in carrier recovery circuits is complex and is discussed in the literature [8].

Every frequency source must be taken into account as well as the associated tracking structures of the communications system. Explicitly simulating every frequency source and all of the associated equipment significantly complicates a simulation. However, this complication can be avoided if our objective is to estimate system-level performance, i.e. BER/SER. In this case, it is not the detailed evolution of the process that we are interested in, but rather the net effect on the decision variable, which is the waveform to be sampled for taking decisions. For this purpose, it is sufficient to define an equivalent process to be injected at the receiver. This equivalent process replaces all of the sources and equipment that produce it. It is assumed that the phase noise contributions of all local oscillators are additive [9]. In order to specify the equivalent noise source we need its pdf, which is also quite difficult to obtain. The relationship between this process and the actual system is not necessarily obvious or simple. Therefore, in order to further simplify the problem, the simulation has been carried out with a static phase noise injected in reception as an upper bound of the maximum phase noise allowed in our system. Having calculated the total permissible phase noise contributions for all local oscillators, it is necessary to allocate a suitable proportion of this total to each source in the phase noise budget.

In the same way, we have studied the degradation of system quality as a function of receiver clock jitter, i.e. as a function of the optimum sample point variation that appears in the synchronization circuits. The previous discussion applies virtually unchanged to the symbol synchronization problem, except that the jitter, instead of being expressed in degrees, is expressed in the time domain (i.e. with reference to a symbol normalized duration).

The simulator used was the SPW (Signal Processing WorkSystem) where the low pass equivalent or complex envelope concept is applied, which allows a hierarchical system description. The BER/SER ratio was estimated by means of the quasianalytical (semianalytic) approach and the Monte Carlo method so that the results could be compared and checked. Monte Carlo simulations allow a precise estimation of the system error rate but they involve a high computational cost, which becomes excessive for low BER/SER values. Therefore, the results obtained from the simulations are not presented for not low values of $\mathrm{BER} / \mathrm{SER}$. However, the tendency suggested by the curves for higher $\mathrm{Eb} / \mathrm{No}$ ratios can clearly be seen.

\subsection{Satellite QPSK subsystem.}

The system block diagram used in the computer simulations is shown in figure 3 . It consists of a random binary source, a QPSK modulator using Gray encoding, a square root raised cosine filter with roll-off equal to 0.35 $(35 \%)$ that includes a $x / \sin x$ equalizer, and the satellite transponder implemented according to 
Saleh's model [10], which provides an excellent fit with the TWT behaviour when four coefficients are used to model the $\mathrm{AM} / \mathrm{AM}$ and $\mathrm{AM} / \mathrm{PM}$ characteristics.

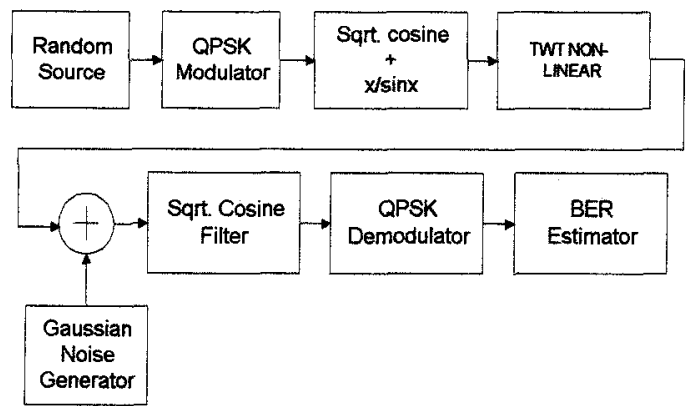

Figure 3. QPSK system block diagram.

White gaussian noise is added to the signal only in the downlink. There are two fundamental reasons for this: first, in satellite communications systems it is the downlink which limits the system performance and, secondly, the presence of noise before non linear blocks (TWT) leads to non gaussian effects which are very difficult to characterize and analyze.

The receiver (TDT) is made up of the corresponding square root raised cosine filter with roll-off 0.35 (to perform wave conformation), the QPSK demodulator and the BER estimation block.

Figure 4 shows, in a simplified form, how the phase noise effect is introduced into the system.

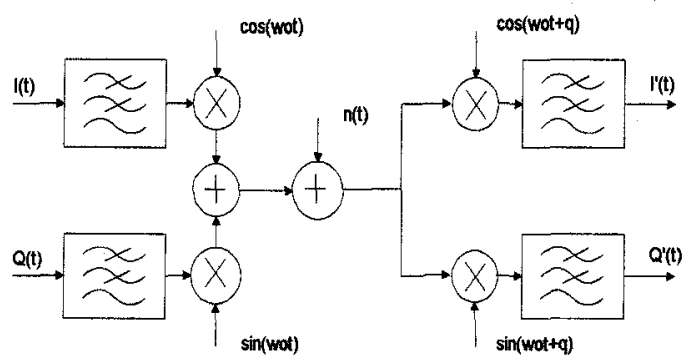

Figure 4. Simplified model of the phase noise effect.

where: $n(t)$ : AWGN.

q: static phase noise.
Figure 5 compares the BER versus $\mathrm{Eb} /$ No curve obtained from the simulations to the theoretical curve for an ideal QPSK. A

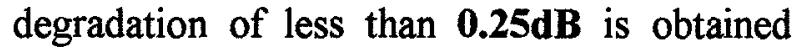
with respect to the ideal QPSK, as is to be expected in a system where the only difference compared with the ideal one is due to the low non-linearity of the transponder and transmitter/receiver filters. This verifies that the simulator functions well and that the estimation of BER is adequate.

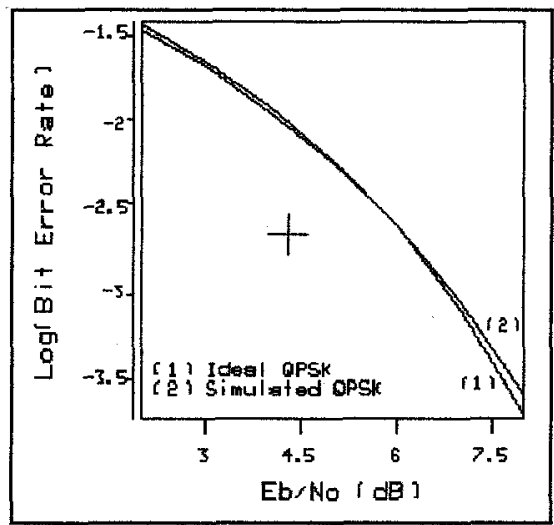

Figure 5. QPSK system: BER evaluation.

Figures 6 and 7 show the behaviour of BER as a function of the phase noise, obtained by simulation. The simulations have been carried out for positive and negative phase noise values. As can be seen in the figures, there is a degradation of the BER when phase noise increases. In this case, the degradation is not critical, i.e. the system quality can be recovered by increasing the $\mathrm{Eb} / \mathrm{No}$ ratio. These curves allow us to determine the highest $\mathrm{Eb} / \mathrm{No}$ degradation allowed in our system, for a fixed BER, which establishes an upper bound for the tolerable phase noise. For example, a $9^{\circ}$ phase noise results in an Eb/No degradation of $1.25 \mathrm{~dB}$ for a fixed BER of $-3 \mathrm{~dB}$. The degradation increases when the $\mathrm{Eb} /$ No ratio is higher, i.e. when our system BER requirements are more demanding.

In figure 8, the results of the simulation are presented showing BER performance versus $\mathrm{Eb} / \mathrm{No}$ ratio, as a function of the sample point in the receiver sample/decide circuit. It can be seen that the optimum sample point is in 
the middle of the symbol position. A $\mathbf{1 0 \%}$ variation in the sample point, for a fixed $\mathrm{Eb} / \mathrm{No}=8 \mathrm{~dB}$, results in a BER degradation of 0.5dB. The degradation increases sharply when the clock jitter increases and also when the $\mathrm{Eb} /$ No ratio increases, i.e., when our system requirements are more demanding.

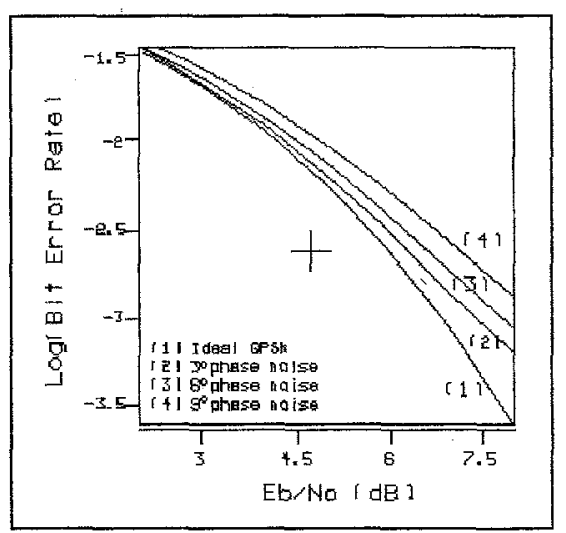

Figure 6. Positive phase noise: BER evaluation.

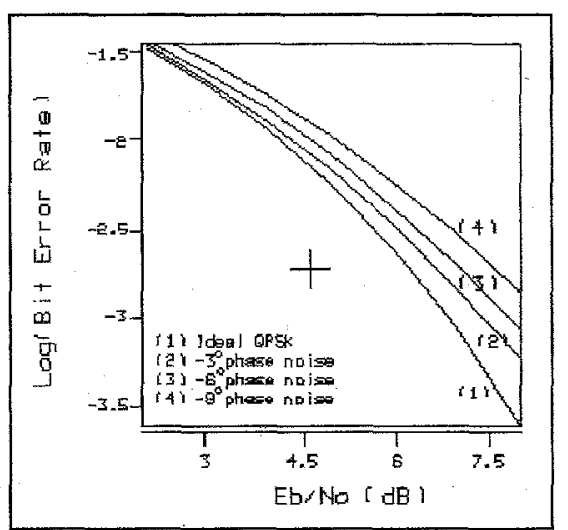

Figure 7. Negative phase noise: BER evaluation.

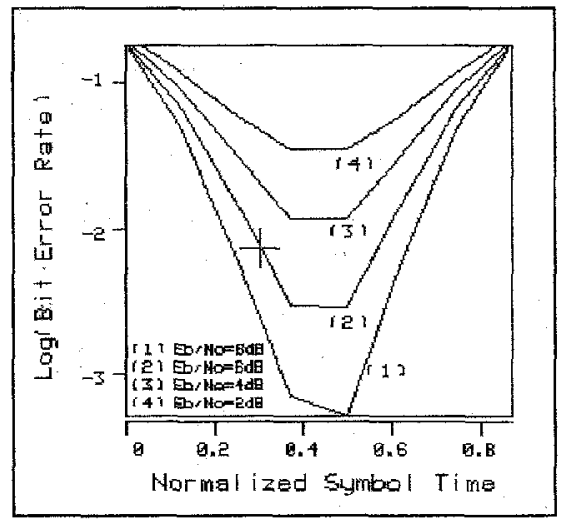

Figure 8. BER performance versus clock jitter as a function of $\mathrm{Eb} / \mathrm{No}$.

\subsection{Cable network 64-QAM subsystem.}

In this case, the system quality is characterized by means of SER. First, in order to isolate phase noise and jitter effects, we simulated the system without considering the SMATV channel. Then, the simulation with the added SMATV channel effects was performed. The simulation results will be presented in the same order.

- SMATV channel characterization: SMATV distribution networks introduce some channel distortion due to mismatching, as a result of the non ideal device behaviour. Distortion can be evaluated by either the frequency response or the time domain impulsive response. The time domain impulsive response is a main impulse followed by delayed and attenuated replicas produced by microreflections among the network elements. In the frequency domain the VHF/UHF band is distorted in a periodic-like shape as a result of adding of several sinusoids with different periods.

SMATV channel characterization is based on statistical evaluation of measurements on a great variety of typical installations and a great number of outlets, summarised in the literature [7].

The system block diagram used in the computer simulations is shown in figure 9 . The transmitter (TDT) consists of a 64-QAM random source (64-QAM modulator) using Gray encoding, a square root raised cosine filter with roll-off equal to $0.15(15 \%)$ that includes a $\mathrm{x} / \sin \mathrm{x}$ equalizer. White gaussian noise is added to the signal and the resulting signal is passed through the SMATV channel. The receiver consists of the corresponding square root raised cosine filter with a roll-off factor of 0.15 to perform wave conformation, the 64-QAM demodulator and the SER estimation block. The SER has been estimated by using a semianalytic approach and the Monte Carlo method for greater verification and comparison of results.

In figure 10 the SER curve versus $\mathrm{Eb} / \mathrm{No}$ ratio, obtained by simulation, is compared to the ideal 64-QAM theoretical curve. For this comparison with the ideal 
response, the simulation has been executed without a channel so that the signal degradation will be as low as possible. As can be seen in the figure, the signal degradation with respect to the theoretical curve is less than $0.5 d B$ in the Eb/No ratio. This confirms that the simulator functions well and that the SER estimation is suitable.

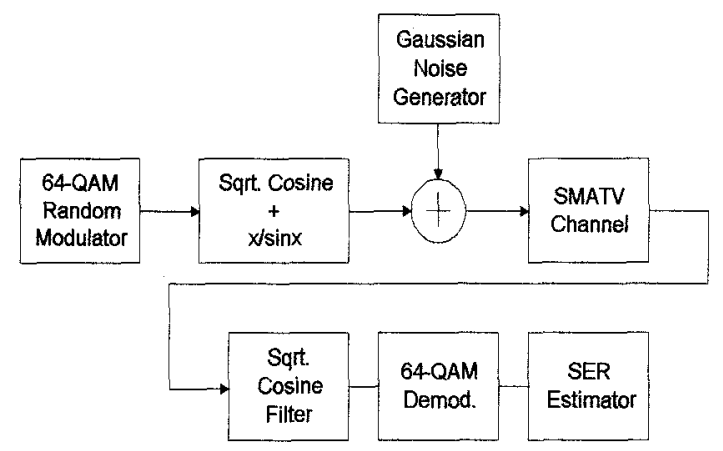

Figure 9. 64-QAM system block diagram.

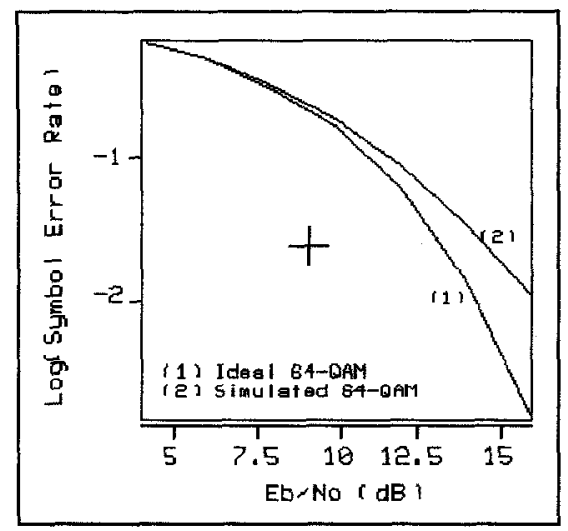

Figure 10. 64-QAM system: SER verification.

The degradation is greater for the 64QAM system than for the QPSK scheme due to the phase distortion of the filters, which causes higher degradation of the QAM signal.

In those cases where receiver static phase noise is added, a degradation in SER performance is observed. Figures 11 and 12 show the results of the simulations. The SER performance is plotted as a function of positive and negative phase noise, respectively.

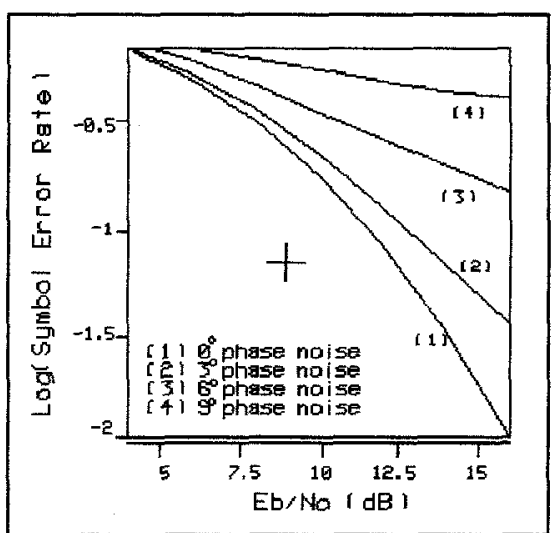

Figure 11. Positive phase noise: SER evaluation.

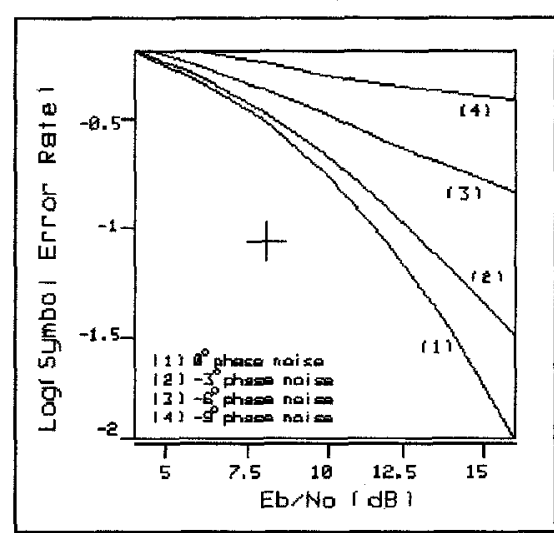

Figure 12. Negative phase noise: SER evaluation.

The degradation becomes critical for static phase noise greater than $\pm 3^{\circ}$, where SER cannot be improved even if the $\mathrm{Eb} / \mathrm{No}$ ratio is increased, i.e. there is saturation. Comparison of these results with the ones obtained in the QPSK simulations shows that the 64-QAM modulation scheme is more sensitive to phase noise. This is a consequence of the higher constellation state numbers, where a small phase noise can produce a symbol error. In QPSK, the symbols are more separated in the constellation and therefore a higher phase noise is needed to cause an error.

Figure 13 shows the SER performance obtained by simulation as a function of the receiver sample position (clock jitter) with respect to the normalized symbol position, versus the $\mathrm{Eb} / \mathrm{No}$ ratio. It can be seen that the optimum sample point is in the middle of the symbol duration. A $10 \%$ sample point devia- 
tion, for a fixed $\mathrm{Eb} / \mathrm{No}=12 \mathrm{~dB}$, causes a $\mathrm{SER}$ degradation of $\mathbf{0 . 6} \mathbf{d B}$, which increases when working at lower error rates.

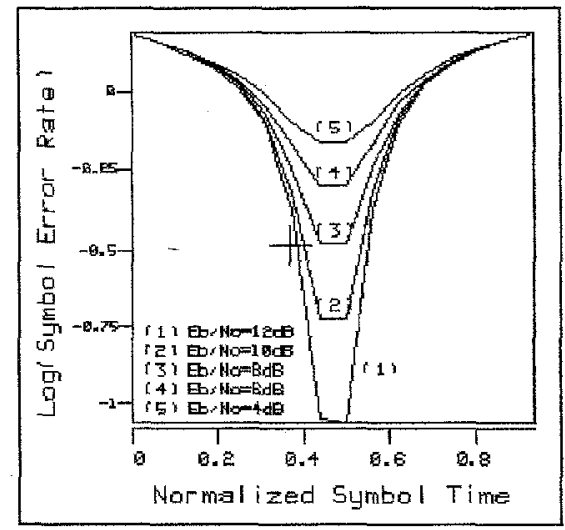

Figure 13. SER performance versus clock jitter as a function of $\mathrm{Eb} / \mathrm{No}$.

In the computer simulations with the SMATV channel, we considered a simplified network model consisting of a principal signal and a single delayed and attenuated echo (i.e. Rummler's model). This simple model provides a general understanding of how the system quality varies as a function of the echo characteristics.

The echo has been delayed one symbol length. For a system using 64-QAM modulation with a symbol rate of $6.96 \mathrm{Mbaud}$ in a $8 \mathrm{MHz}$ channel and allowing a bit rate higher than $38 \mathrm{Mbit} / \mathrm{s}$, this implies a symbol length of $144 \mathrm{~ns}$. Thus, a highly unfavourable case has been considered because the delay associated with the most important echoes in typical SMATV networks is less than $100 \mathrm{~ns}$. The echo attenuation is varied to observe the effect on SER performance.

Figure 14 shows SER performance versus $\mathrm{Eb} / \mathrm{No}$ ratio, as a function of the echo level when there is a variation from -14 to $-22 \mathrm{~dB}$ and for an assumed fixed delay of one symbol length (144ns).

As can be seen in this figure, obtained by simulation, echoes attenuated by less than $18 \mathrm{~dB}$ produce a critical degradation in system quality, i.e. SER performance cannot be improved even if the $\mathrm{Eb} / \mathrm{No}$ ratio is increased. However, echoes attenuated by more than
$24 \mathrm{~dB}$ are negligible because they produce a degradation in the $\mathrm{Eb} / \mathrm{No}$ ratio of no more than 1.5dB.

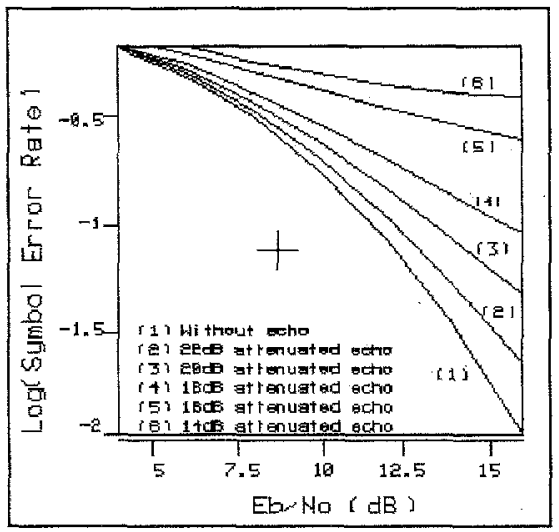

Figure 14. SER performance versus echo level.

Bearing in mind that the amplitude /frequency channel response is a function of the echo level, we can conclude that when echoes are present in the channel, 64-QAM modulation is very sensitive to amplitude distortions. Therefore, high level echoes require equalization to compensate for signal impairment. The equalization function will depend on the number of echoes and also on their level.

In the same way, receiver equalization reduces SER performance degradation resulting from phase noise, allowing us to relax the system requirements.

\section{CONCLUSIONS.}

The results obtained by means of computer simulation show BER/SER performance degradation as a function of phase noise and clock jitter in the two subsystems of the SMATV-DTM system. These results indicate that the QPSK system is less sensitive to phase noise and clock jitter than the QAM system.

Phase noise in the QPSK system, does not result in a critical degradation, i.e. we can recover the BER performance by increasing the $\mathrm{Eb} / \mathrm{No}$ ratio. However, the degradation, and the increase of the transmission power, will be 
system limited, which sets a limit on the maximum phase noise allowed in the system.

In the 64-QAM system, phase noise produces critical degradation: a phase error of only $3^{\circ}$ produces a total loss of system quality. We can conclude, therefore, that this system is very sensitive to phase noise. Similarly, SMATV channel echoes also produce a total loss of system quality, showing that QAM modulation is quite sensitive to amplitude distortion. Consequently, high level echoes require equalization to compensate for signal degradation, allowing system requirements to be relaxed.

\section{REFERENCES}

[1] "HD-SAT: HDTV broadcasting over Ka-band satellite, cable and MMDS networks". Signal Processing of HDTV, VI. Proceedings of the International Workshop on HDTV'94. 26-28 Oct. 1994.

[2] "Broadcast TV: which way to the digital future". Electrotechnology (UK). Vol. 6. No4. (Aug.-Sept. 1995).

[3] "The DVB satellite, cable and SMATV systemswhy the technical choices were made". EBU Tech. Rev. $\mathrm{N}^{\circ}$ 266. p. 24-28.

[4] "Community antenna installations for TV reception". Electron. Telecommun. Vol. 44. No 2-3. p.59-67. (Dec. 1995).

[5] "Direct distribution of satellite digital TV signals in community antenna installations". Electron. Telecommun. Vol. 44. N ${ }^{\circ}$ 2-3. p.68-74. (Dec. 1995).

[6] DIGISMATV Race Project. Satellite Digital TV in Collective Antenna Systems. The DVB-SMATV System. Issue 2.0. Oct. 1995.

[7] "Computer simulations of DVB satellite signals distributed in community receiving installations". Electtron. Telecommun. Vol. 44. $\mathrm{N}^{\mathrm{o}}$ 2-3. p.75-87. (Dec. 1995).

[8] W.P. Robins, "Phase Noise in Signal Sources (Theory and Applications)". IEE Telecommunications Series, 9.
[9] Michel C. Jeruchim, Philip Balaban and K.Sam Shanmugan, "Simulation of Communication Systems". 1992 Plenum Press, New York.

[10] A.A.M. Saleh, "Frecuency-Independent and Frecuency-Dependent Nonlinear Models of TWT Amplifiers". IEE Transactions on Communications, 1981.

\section{Biographies}

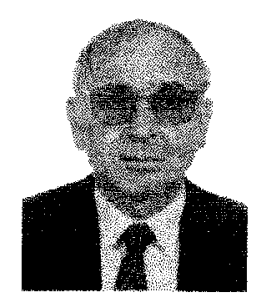

Jose Luís García García (M'85) was born in Zaragoza, Spain, in 1938. He received an M.S.E degree from the University of Zaragoza in 1964 and $\mathrm{PhD}$ from the University of Valladolid, Spain, in 1971. From 1966 to 1973 he was Associate Professor at the University of Valladolid where he worked on analog simulation of systems and the generation of pseudo-random signals. In 1973 he became professor of Electronics Engineering of the Department of Electronics of the University of Cantabria in Santander (SPAIN) where he has been dean of the Telecommunications Engineering School and head of Department. At present he is the head of the Department of Communications Engineering. $\mathrm{He}$ teaches Radiocommunication Systems and Satellite Communications. He has worked in microwave and millimetric wave systems and components for mobile, radiolinks and satellite communications. His current research interests include broadcasting of digital TV through satellite and SMATV-DTM systems; wireless CDMA-SS for indoor applications; low speed CDMA satellite communications and cellular access to broadband services and interactive television at millimetric waves. He is a member of the Committee of the E-12 professional group of the IEEE.

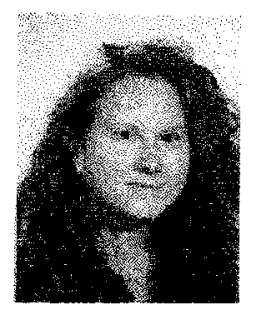

Ana García Armada was born in Santiago de Compostela on 17 June, 1970. She received a degree in Telecommunications Engineering from the ETSIT, Technical University of Madrid in July 1994. Since November 1994 she has been working on Simulation and Rapid Prototyping of the European Digital TV Terrestrial Broadcasting System (dTTb) based on COFDM signals as a $\mathrm{PhD}$ student in the same University. She collaborated in European RACE projects and worked in TIDSA and the European Space Agency-ESTEC during the final years of her undergraduate studies. 
Sergio Merchán González was born in Burgos (SPAIN) on 2 September, 1973. In 1995 he joined the Department of Communications Engineering of the University of Cantabria where he collaborated in a European RACE project, DIGISMATV. He was awarded a degree in Telecommunications Engineering by the University of Cantabria in 1996. Since October 1996, as a PhD student in the same University, he has been participating in another European project, CABSINET, where he is defining and simulating a digital cellular access network to broadband interactive services following the DVB-MS standard. 